\title{
Electric Field-Driven Point Defect Pile-up Near ZnO Polar Surfaces
}

\author{
Prashun Gorai ${ }^{\mathrm{a}}$, Edmund G. Seebauer ${ }^{\mathrm{a}}$ \\ ${ }^{a}$ Department of Chemical and Biomolecular Engineering, University of Illinois, Urbana, IL 61801, USA
}

\begin{abstract}
Electric fields in near-surface space charge regions influence the spatial distribution of charged defects, with implications for the efficiency of photocatalysts and gas sensors. Through isotopic oxygen diffusion experiments on both Zn-terminated $\mathrm{ZnO}$ (0001) and O-terminated $\mathrm{ZnO}$ (0001), the present work better delineates the quantitative relationship of defect pile-up to the degree and direction of near-surface band bending, the rate of defect injection, and the temperature. The two terminations exhibit dramatic differences in behavior from each other, especially in the temperature dependence. The results suggest that the amount of fixed charge at the surface directly affects the injected flux.
\end{abstract}

Keywords: $\mathrm{ZnO}$, defect diffusion, space charge, polar surface

The spatial distribution of native point defects and substitutional dopants near solid-gas and solidsolid boundaries of semiconductors affects the operational efficiency of photovoltaic $[1,2]$, photocatalytic $[3,4]$, and microelectronic $[5,6,7]$ devices. During fabrication steps that require heating, dopant atom segregation or precipitation [8] can occur due to a combination of misfit elastic strain [9], chemical potential gradient $[10,11,12]$ and electrostatic drift $[10,13,14]$. Pile-up of native defects is also possible, and those associated with energy levels deep in the bandgap usually affect device performance adversely [15]. The published literature has focused primarily on electrostatic mechanisms $[10,13,14]$ by which fixed charge on surfaces influences the spatial distribution of nearby charged defects. This laboratory has reported [14] experimental evidence for a distinct mechanism involving field-induced changes in the motion of rapidly mobile defects such as interstitials, coupled with their exchange into a station-

Email address: eseebaue@illinois.edu (Edmund G. Seebauer)

Preprint submitted to Solid State Ionics ary reservoir such as the host crystal lattice. Subsequent modeling demonstrated [16] that these effects enhance or diminish the concentration of the mobile defects within the surface space charge region (SCR), leading to a pile-up or depletion of the corresponding species exchanged into the reservoir - depending upon the direction of the field and the sign of the defect's charge.

The experimental results underlying that work involved ${ }^{18} \mathrm{O}_{2}$ gas exchange with rutile $\mathrm{TiO}_{2}(110)$, wherein pile-up of substitutional isotopic oxygen was observed within several nanometers of the surface. However, the results were not sufficiently precise to establish the quantitative relationship of pile-up to the degree and direction of near-surface band bending, the rate of defect injection, or the temperature. The present work employs similar experimental methods with $c$-axis $\mathrm{ZnO}$, both Zn-terminated (0001) and O-terminated $(000 \overline{1})$, in tandem with the mathematical model [16], to examine these relationships. The two terminations exhibit dramatic differences in behavior from each other, especially in the temperature dependence. Taken together, the results suggest that the amount of fixed charge at the surface may

(C) 2016. This manuscript version is made available under the Elsevier user license

http://www.elsevier.com/open-access/userlicense/1.0/ 


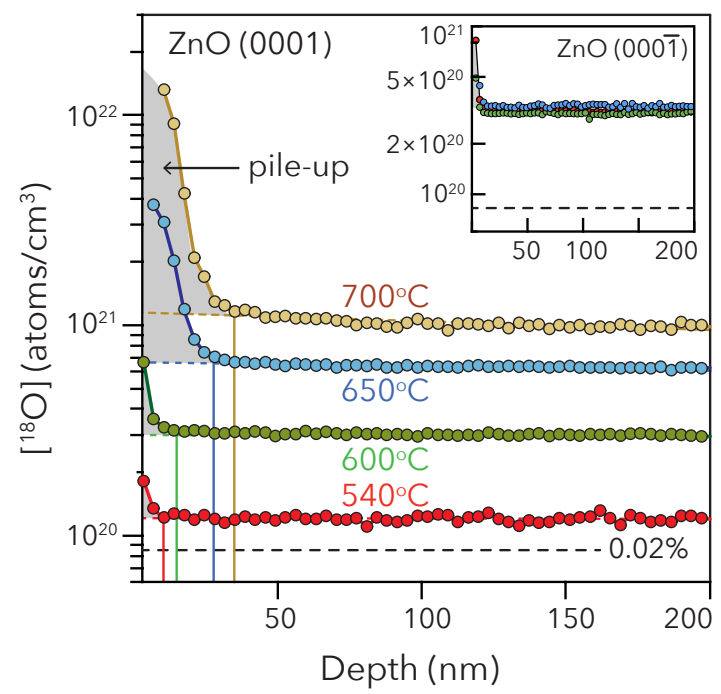

Figure 1: Isotopic-labeled oxygen $\left({ }^{18} \mathrm{O}\right)$ diffusion profiles in $\mathrm{Zn}-\mathrm{ZnO}$ (and $\mathrm{O}-\mathrm{ZnO}$ inset) samples annealed in ${ }^{18} \mathrm{O}_{2}$ gas $\left(5 \times 10^{-5}\right.$ Torr $)$ at $540^{\circ} \mathrm{C}, 600^{\circ} \mathrm{C}, 650^{\circ} \mathrm{C}$ and $700^{\circ} \mathrm{C}$ for 105 min. The near-surface profile shows pile-up of the isotopic marker within $\sim 10-35 \mathrm{~nm}$ of the surface. The exponential profile beyond the pile-up zone represents bulk diffusion.

exert a direct influence on the injected flux.

As discussed previously [14, 16], mobile defects formed at the surface and injected into the bulk undergo Fickian diffusion. For downward band bending in the SCR of natively $n$-type materials such as $\mathrm{TiO}_{2}$ and $\mathrm{ZnO}$, the near-surface field $\epsilon(x)$ points into the bulk, which induces field drift of negatively charged defects such as $\mathrm{O}_{i}^{2-}$ toward the surface and impedes migration into the bulk. The prolonged residence time of these defects in the SCR increases the degree to which they exchange with the reservoirs that exist there, such as the lattice via kick-in. In an isotopic oxygen $\left({ }^{18} \mathrm{O}\right)$ gas-solid exchange experiment, the labeled $\mathrm{O}$ marker replaces the existing lattice $\mathrm{O}$ over time. Since ${ }^{18} \mathrm{O}$ and ${ }^{16} \mathrm{O}$ are chemically alike, they are thermodynamically interchangeable in the lattice; the ${ }^{18} \mathrm{O}$ represents an isotopic rather than a chemical defect. The resultant pile-up (or valley) can be measured with secondary ion mass spectrometry (SIMS).

The present experiments employed an apparatus described previously [14]. Single crystals of wurtzite
$\mathrm{ZnO}$ with polar (0001) Zn-terminated $(\mathrm{Zn}-\mathrm{ZnO})$ or $(000 \overline{1})$ O-terminated $(\mathrm{O}-\mathrm{ZnO})$ surfaces were exposed to isotopically-labeled oxygen gas $\left({ }^{18} \mathrm{O}_{2}\right)$ at elevated temperatures $\left(540-700^{\circ} \mathrm{C}\right)$ under O-rich conditions $\left(\mathrm{P}_{\mathrm{O}_{2}} \sim 5 \times 10^{-5}\right.$ Torr $)$ for $105 \mathrm{~min}$. Before isotopic exposure, the specimens were degreased ex situ as described in Ref. 14, and then heated in natural abundance $\mathrm{O}_{2}$ to equilibrate the native defect concentrations at the same temperature and pressure as the subsequent isotopic exposure. After that exposure, ${ }^{18} \mathrm{O}$ concentration profiles were measured ex situ with time-of-flight SIMS using a PHI-TRIFT III instrument [14] and electron flooding. To ensure that the near-surface profiles are not complicated by measurement artifacts [17], low depth profiling rates were employed and data points at or very near the surface were discarded (Figure 1). Additionally, multiple measurements were performed at different spots on the sample to check for reproducibility of the pileup profile. Special care was taken to maintain instrumental base pressures that minimized mass interferences from $\mathrm{H}_{2}{ }^{16} \mathrm{O}$, which has a mass similar to ${ }^{18} \mathrm{O}$. In Figure 1, the near-surface profiles change dramatically and systematically with annealing conditions. If the existence of pile-up were an artifact of the SIMS measurements, the annealing conditions would not exert any systematic effect of on the profiles.

Figure 1 (and inset) shows example ${ }^{18} \mathrm{O}$ diffusion profiles for $\mathrm{Zn}-\mathrm{ZnO}$ (and $\mathrm{O}-\mathrm{ZnO}$ ). The profiles qualitatively resemble those obtained in rutile $\mathrm{TiO}_{2}(110)$ [14, 16], with two distinct regimes:(1) near-surface $(\sim 10-35 \mathrm{~nm})$ pile-up of the isotopic O marker, and (2) deeper profile extensions into the bulk. Unlike $\mathrm{TiO}_{2}$, for which pile-up was typically small, shallow $(<10 \mathrm{~nm})$ and poorly reproducible, $\mathrm{Zn}-\mathrm{ZnO}$ exhibits pile-up that is often large, deeper (up to $35 \mathrm{~nm}$ ), and straightforward to reproduce among various specimens. O-ZnO exhibits smaller and shallower pile-up, but the reproducibility is still good. As in the case of $\mathrm{TiO}_{2}$, the profiles in the deeper bulk $(>35 \mathrm{~nm})$ have very shallow, but non-zero, slopes. The migration of isotopic marker into the bulk is perhaps more evident in Figure 1 from the large increase in overall ${ }^{18} \mathrm{O}$ concentration throughout the entire deep bulk. Extensive analysis of this section of the profile has been reported elsewhere [18], and is not a focus of 
this work except where noted below for the purposes of near-surface profile analysis.

Previous work [14] has quantified the amount of pile-up $(P)$ as the total integrated area between the near-surface pile-up profile and the deep-bulk profile extrapolated to the surface, shown as shaded regions in Figure 1. For this extrapolation, an exponential function was used (linear on the semilogarithmic scale of Figure 1) as in the case of $\mathrm{TiO}_{2}[14,16]$. The width of the pile-up region for a given profile was determined as the depth at which the profile begins to deviate substantially from the exponential function used for extrapolation. These widths are marked in Figure 1 as vertical lines. For $\mathrm{Zn}-\mathrm{ZnO}$, both this width and $P$ itself increase with temperature from $540^{\circ} \mathrm{C}$ to $700^{\circ} \mathrm{C}$. For $\mathrm{O}-\mathrm{ZnO}$, the width and $P$ both remain roughly constant with increasing temperature. Regardless of temperature or termination, $P$ accounts for $<1 \%$ of the total number of ${ }^{18} \mathrm{O}$ injected into the bulk - corresponding to the behavior in rutile $\mathrm{TiO}_{2}$ [14]. In this small-perturbation limit, it is a good mathematical approximation [16] to analyze the pile-up and deeper-bulk regions separately.

Substantial evidence suggests that the oxygen interstitial $\mathrm{O}_{i}$ mediates oxygen diffusion in $\mathrm{TiO}_{2}$ under clean-surface conditions [14]. That evidence includes the elevated magnitude of the observed $\mathrm{O}$ diffusivity compared to the literature, the exponential (rather than error-function) shapes of the profiles, the existence and behavior of near-surface pile-up [14], and the predictions of quantum calculations[19] that $\mathrm{O}_{i}^{2-}$ is thermodynamically more stable than other Orelated native defects. The analogous body of published evidence for $\mathrm{ZnO}$ is smaller, and rests mainly on quantum calculations $[20,21]$ and diffusion experiments [18] that point to $\mathrm{O}_{i}^{2-}$ as the most stable O-related native defect.

The $\mathrm{ZnO}$ used in this work is almost certainly $n$ type because of incorporated hydrogen donors [22, 23], with the charge density set by the hydrogen concentration. The existence of pile-up in the profiles of Figure 1 (contrasted with a "valley") indicates an electric field directed opposite to the diffusional flux, which in $n$-type material implies the existence of an accumulation-type space charge region with downward band bending [24]. Our previously-developed
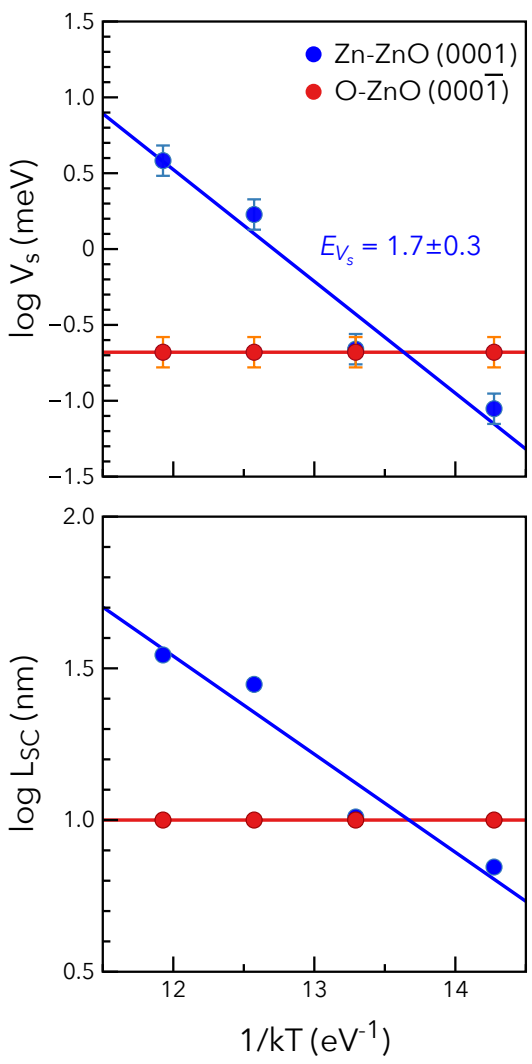

Figure 2: (a) Arrhenius temperature dependence of $V_{s}$ for the $\mathrm{Zn}-\mathrm{ZnO}$ (red) and $\mathrm{O}-\mathrm{ZnO}$ (blue) at $5 \times 10^{-5}$ Torr. The activation energy for buildup of $\mathrm{V}_{s}$ is $\sim 1.7 \mathrm{eV}$ for the $\mathrm{Zn}-\mathrm{ZnO}$ surface. $V_{s}$ remains almost constant at the O-ZnO surface. (b) Widening of the pile-up width $\left(L_{S C}\right)$ with temperature for $\mathrm{Zn}-\mathrm{ZnO}$ and $\mathrm{O}-\mathrm{ZnO}$ surfaces.

analytical expressions [16] can be used to estimate the surface potential $V_{s}$ from the surface concentration $C_{S, 1}$, using the equation:

$$
C_{S, 1}-\left.C_{S 0}\right|_{x=0}=\frac{F t}{\lambda} \chi_{1}
$$

where $F$ is the flux of mobile species injected at the surface, $\lambda$ the mean diffusion length of that species before exchange with the reservoir, $C_{S 0}$ the background concentration of natural abundance marker in the material, $t$ the diffusion time, and $\chi_{1}$ a dimensionless factor. Extrapolation of the deep bulk profile to the surface yields a hypothetical zero-field 
concentration equal to $F t / \lambda$ [16]. For drift directed oppositely to diffusion, the surface concentration of marker is always enhanced by a factor of $\chi_{1}$. With $\lambda$ and $F$ calculated from the deep bulk profile, $\chi_{1}$ can be calculated from Eq. (1). From $\chi_{1}$, the magnitude of the surface electric field $\epsilon(x)$ can be determined from the relation [16]:

$$
\chi_{1}=\sqrt{\left(\frac{\alpha \epsilon_{1} \lambda}{2}\right)^{2}+1}-\frac{\alpha \epsilon_{1} \lambda}{2}
$$

where $\alpha=q / k_{B} T, q$ is the charge on the mobile species, and $k_{B}$ is Boltzman's constant. The value of $\epsilon_{1}$ permits the estimation of the surface potential $V_{s}$ from classical electrostatic relations involving the background carrier concentration of the material and its dielectric constant. The pile-up region has approximately the same width $L_{S C}$ as the SCR. Evidence that the donor concentration remains constant with $\mathrm{T}$ comes from the fact that both $L_{S C}$ and $\chi_{1}$ remain independent of $\mathrm{T}$ for $\mathrm{O}-\mathrm{ZnO}$.

Fitting an Arrhenius temperature dependence to $\lambda$ for the $\mathrm{Zn}-\mathrm{ZnO}$ surface yields a pre-exponential factor of $105.36 \pm 0.73 \mathrm{~nm}$ and an effective activation energy $E_{\lambda}=0.28 \pm 0.13 \mathrm{eV}$. A similar procedure for $F$ yields an activation energy $E_{\text {Flux }}=1.67 \pm 0.32 \mathrm{eV}$ [14]. For O-ZnO, $E_{\lambda}$ is similar in magnitude to that of $\mathrm{Zn}-\mathrm{ZnO}$, while $E_{\mathrm{Flux}}$ is roughly T-independent. The similar values of $E_{\lambda}$ for $\mathrm{Zn}-\mathrm{ZnO}$ and O-ZnO point to the same $\mathrm{O}$ diffusion species and mechanism in both cases.

With the assumption of a -2 charge state for $\mathrm{O}_{i}$, Eqs. (1) and (2) may be applied to the profiles of Figure 1 to yield $V_{s}$ as a function of temperature, as shown in Figure 2(a). The magnitude of $V_{s}$ in Figure $2(\mathrm{a})$ is on the order of only a few $\mathrm{meV}$, which highlights the sensitivity of the near-surface pile-up profile to built-in electric fields in the SCR. Conventional measurement techniques such as Kelvin probe microscopy are not sensitive enough to resolve surface potentials of the order of few $\mathrm{meV}$ at most [25]. At the typically high temperatures used in the diffusion experiments, thermalization of charge carriers tends to significantly flatten band bending in the $\mathrm{SCR}$, which can explain the small values of $V_{s}$ in Figure $2(\mathrm{a})$.

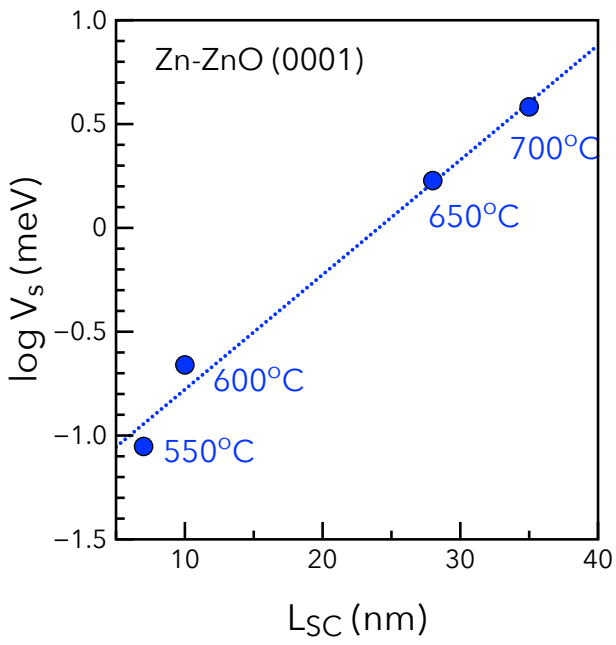

Figure 3: A linear fit to a semi-logarithmic plot of surface potential $\left(V_{s}\right) v s$. pile-up width $\left(L_{S C}\right)$ confirms that the widening of the space charge region with temperature is in response to the increasing $V_{s}$ in an accumulation-type space charge.

On the $\mathrm{Zn}-\mathrm{ZnO}$ surface, $V_{s}$ increases exponentially by almost an order of magnitude from $\sim 0.1 \mathrm{meV}$ at $540^{\circ} \mathrm{C}$ to $\sim 3.8 \mathrm{meV}$ at $700^{\circ} \mathrm{C}$. The activation energy $E_{V_{s}}$ for the variation of $V_{s}$ is $1.7 \pm 0.3 \mathrm{eV}$ with a pre-exponential factor of $109.4 \pm 1.6 \mathrm{meV}$. For O$\mathrm{ZnO}$, the pile-up profile does not appreciably change with temperature so $V_{s}$ is also roughly constant with temperature. Figure 2(b) shows the temperature dependence of $L_{S C}$. The value remains constant near $10 \mathrm{~nm}$ for $\mathrm{O}-\mathrm{ZnO}$; it increases exponentially with $\mathrm{T}$ for $\mathrm{Zn}-\mathrm{ZnO}$ - similar to the $V_{s}$ buildup. Note that the electric field in an accumulation region decays exponentially in space, as opposed to quadratic decay in a depletion region [18]. For an accumulation-type space charge region, a semi-logarithmic plot of $V_{s}$ versus $L_{S C}$ should therefore be approximately linear as contrasted with quadratic for a depletion region. The linearity of a semi-logarithmic plot of $V_{s}$ versus $L_{S C}$ for the Zn-ZnO surface, shown in Figure 3, confirms the exponential functional form and thereby the existence of an accumulation region as discussed above. This confirmation gives confidence that the mathematical model derived in Ref. 16 accurately describes the behavior of pile-up in its various aspects. 
The correspondence in the general behavior of $E_{V_{s}}$ and $E_{\text {Flux }}$ for $\mathrm{Zn}-\mathrm{ZnO}$ and $\mathrm{O}-\mathrm{ZnO}$ is notable. The presence of the accumulation-type space charge in an $n$-type semiconductor implies the presence of excess electrons in the SCR compared to the bulk, which in conjunction with polar effects [18] could be responsible for enhancing the adsorption and dissociation of $\mathrm{O}_{2}$ as well as the subsequent charging of the injected $\mathrm{O}$ interstitial species. Indeed, recent first-principles calculations [26] highlight the propensity of adsorbed $\mathrm{O}$ and $\mathrm{O}_{2}$, as well as $\mathrm{O}_{i}$, to soak up nearby electrons even when no excess is available.

That such an influence of $V_{s}$ on $F$ is plausible may be established as follows. From the context of gas sensors, adsorption of $\mathrm{O}_{2}$ is already known to withdraw electrons from $\mathrm{ZnO}$ and thereby affect $V_{s}$ [27]. It is therefore reasonable to suppose that, for surfaces wherein the surface Fermi energy is pinned, the value of $V_{s}$ and the corresponding availability of conduction-band electrons could influence the amount of $\mathrm{O}_{2}$ (or $\mathrm{O}$ ) adsorption. As adsorption is the first step in the kinetic sequence leading to $\mathrm{O}_{i}$ injection, changes in the amount of injectable oxygen at the surface would translate into changes in $F$. Similarly, the value of $V_{s}$ in some semiconductors affects the average charge state of near-surface point defects whenever an ionization level is favorably situated within the band gap $[5,16]$. The ionization levels for $\mathrm{O}_{i}$ in $\mathrm{ZnO}$ are not known, although the possibility of multiple charge states has not been excluded.

Evidence for the influence of $V_{s}$ on $F$ may be found in the fact that $V_{s}$ and $L_{S C}$ increase with temperature for $\mathrm{Zn}-\mathrm{ZnO}$, which increases the concentration of electrons in the SCR and also leads to increased O injection flux [18]. Correspondingly, $V_{s}$ and $L_{S C}$ remain constant with temperature for $\mathrm{O}-\mathrm{ZnO}$, as does the injected flux. The differences in behavior between $\mathrm{Zn}-\mathrm{ZnO}$ and $\mathrm{O}-\mathrm{ZnO}$ undoubtedly arise from the distinct surface reconstructions and atomic geometries for the two terminations [26]. However, we do not ascribe significance to the exact numerical correspondence between $E_{V_{s}}$ and $E_{\text {Flux }}$. $E_{\text {Flux }}$ for $\mathrm{Zn}-\mathrm{ZnO}$ has been linked directly to the elementary-step injection barrier for $\mathrm{O}_{i}$, while for O-ZnO, $E_{\mathrm{Flux}}$ appears to represent a balance between the injection barrier and the concentration of injectable oxygen. The physical mechanism for a direct correspondence between $E_{V_{s}}$ and $E_{\text {Flux }}$ is not clear.

The findings of this work give important confirmation of the mathematical treatment of Ref. 16 as a basis for interpreting pile-up in isotopic exchange experiments, and also suggest that the amount of fixed charge at the surface exert a direct influence on the injected flux. This coupling is likely to be more pronounced for polar semiconductor surfaces than for nonpolar, as the need for stabilization mechanisms to avoid thermodynamic instability $[28,29,30,31]$ are more likely to support charged oxygen species that facilitate injection into the bulk as charged $\mathrm{O}_{i}$. For cases in which direct control over the spatial distribution of near-surface point defects is desired, this coupling between fixed charge and flux may complicate the task.

\section{Acknowledgments}

This work was partially supported by the University of Illinois College of Engr. Strategic Research Initiatives program and National Science Foundation (DMR 10-05720, DMR 13-06822). SIMS was performed at the Center for Microanalysis of Materials at the University of Illinois at Urbana-Champaign. P.G. acknowledges the fellowship support from the Dow Chemical Company.

[1] Michael Gratzel. Photoelectrochemical cells. Nature, 414:338, 2001.

[2] Hannes Wagner, Amir Dastgheib-Shirazi, Byungsul Min, Ashley E. Morishige, Michael Steyer, Giso Hahn, Carlos del Caizo, Tonio Buonassisi, and Pietro P. Altermatt. Optimizing phosphorus diffusion for photovoltaic applications: Peak doping, inactive phosphorus, gettering, and contact formation. J. Appl. Phys., 119(18):185704, 2016.

[3] Amy L. Linsebigler, Guangquan Lu, and John T. Yates. Photocatalysis on $\mathrm{TiO}_{2}$ surfaces: Principles, mechanisms, and selected results. Chem. Rev., 95:735-758, 1995. 
[4] Antonino Gulino, Guglielmo G. Condorelli, Ignazio Fragal, and Russell G. Egdell. Surface segregation of $\mathrm{Sb}$ in doped $\mathrm{TiO}_{2}$ rutile. Appl. Surf. Sci., 90(3):289-295, 1995. ISSN 0169-4332.

[5] Michael Y. L. Jung, Rudiyanto Gunawan, Richard D. Braatz, and E. G. Seebauer. Effect of near-surface band bending on dopant profiles in ion-implanted silicon. J. Appl. Phys., 95:1134, 2004.

[6] S. Tsui, A. Baikalov, J. Cmaidalka, Y. Y. Sun, Y. Q. Wang, Y. Y. Xue, C. W. Chu, L. Chen, and A. J. Jacobson. Field-induced resistive switching in metal-oxide interfaces. Appl. Phys. Lett., 85:317, 2004.

[7] A. Maiti, M. F. Chisholm, S. J. Pennycook, and S. T. Pantelides. Dopant segregation at semiconductor grain boundaries through cooperative chemical rebonding. Phys. Rev. Lett., 77:13061309, Aug 1996.

[8] Joachim Maier. Ionic conduction in space charge regions. Prog. Solid State Chem., 23(3):171, 1995.

[9] Wonyoung Lee, Jeong Woo Han, Yan Chen, Zhuhua Cai, and Bilge Yildiz. Cation size mismatch and charge interactions drive dopant segregation at the surfaces of manganite perovskites. J. Amer. Chem. Soc., 135:7909, 2013.

[10] Jeri Ann S. Ikeda and Yet-Ming Chiang. Space charge segregation at grain boundaries in titanium dioxide: I, relationship between lattice defect chemistry and space charge potential. J. Amer. Ceram. Soc., 76:2437, 1993.

[11] J. Jamnik and J. Maier. Charge transport and chemical diffusion involving boundaries. Solid State Ionics, 94:189, 1997.

[12] L.J. Brillson, Y. Dong, D. Doutt, D.C. Look, and Z.-Q. Fang. Massive point defect redistribution near semiconductor surfaces and interfaces and its impact on schottky barrier formation. Physica B: Condens. Matter, 404:4768, 2009.
[13] Roger A. De Souza and Manfred Martin. Using ${ }^{18} \mathrm{O} /{ }^{16} \mathrm{O}$ exchange to probe an equilibrium space-charge layer at the surface of a crystalline oxide: method and application. Phys. Chem. Chem. Phys., 10:2356, 2008.

[14] Prashun Gorai, Alice G. Hollister, and Edmund G. Seebauer. Electrostatic drift effects on near-surface defect distribution in $\mathrm{TiO}_{2}$. Appl. Phys. Lett., 103(14):141601, 2013.

[15] E. Kask, T. Raadik, M. Grossberg, R. Josepson, and J. Krustok. Deep defects in $\mathrm{Cu}_{2} \mathrm{ZnSnS}_{4}$ monograin solar cells. Energy Procedia, 10:261$265,2011$.

[16] Prashun Gorai and Edmund G. Seebauer. Kinetic model for electric-field induced point defect redistribution near semiconductor surfaces. Appl. Phys. Lett., 105(2):021604 1-5, 2014.

[17] Sarah Fearn, Jeremy Rossiny, and John Kilner. SIMS artifacts in the near surface depth profiling of oxygen conducting ceramics. Solid State Ionics, 179(2126):811 - 815, 2008. ISSN 01672738. Solid State Ionics 16: Proceedings of the 16th International Conference on Solid State Ionics (SSI-16), Part I.

[18] Prashun Gorai, Elif Ertekin, and Edmund G. Seebauer. Surface-assisted defect engineering of point defects in ZnO. Appl. Phys. Lett., 108: 241603, 2016.

[19] Sutassana Na-Phattalung, M. F. Smith, Kwiseon Kim, Mao-Hua Du, Su-Huai Wei, S. B. Zhang, and Sukit Limpijumnong. Firstprinciples study of native defects in anatase $\mathrm{TiO}_{2}$. Phys. Rev. B, 73:125205, 2006.

[20] Gui-Yang Huang, Chong-Yu Wang, and JianTao Wang. First-principles study of diffusion of oxygen vacancies and interstitials in $\mathrm{ZnO}$. J. Phys. Condens. Mat., 21(19):195403, 2009.

[21] Paul Erhart and Karsten Albe. First-principles study of migration mechanisms and diffusion of oxygen in zinc oxide. Phys. Rev. B, 73:115207, 2006. 
[22] E. V. Lavrov, F. Herklotz, and J. Weber. Identification of two hydrogen donors in $\mathrm{ZnO}$. Phys. Rev. B, 79:165210, 2009.

[23] Chris G. Van de Walle. Hydrogen as a cause of doping in zinc oxide. Phys. Rev. Lett., 85: 1012-1015, 2000.

[24] Hans Lüth. Space-Charge Layers at Semiconductor Inferfaces, pages 323-376. Springer Berlin Heidelberg, Berlin, Heidelberg, 2010. ISBN 978-3-642-13592-7.

[25] T. Ouisse, F. Martins, M. Stark, S. Huant, and J. Chevrier. Signal amplitude and sensitivity of the kelvin probe force microscopy. Appl. Phys. Lett., 88(4):043102, 2006.

[26] Prashun Gorai, Edmund G. Seebauer, and Elif Ertekin. Mechanism and energetics of $\mathrm{O}$ and $\mathrm{O}_{2}$ adsorption on polar and non-polar $\mathrm{ZnO}$ surfaces. J. Chem. Phys., 144:184708, 2016.

[27] G. Eranna, B. C. Joshi, D. P. Runthala, and R. P. Gupta. Oxide materials for development of integrated gas sensors: a comprehensive review. Critical Rev. Solid State Mater. Sci., 29: 111, 2004.

[28] Jacek Goniakowski, Fabio Finocchi, and Claudine Noguera. Polarity of oxide surfaces and nanostructures. Rep. Prog. Phys., 71(1):016501, 2008.

[29] Claudine Noguera. Polar oxide surfaces. J. Phys. Condens. Matt., 12(31):R367, 2000.

[30] Georg Kresse, Olga Dulub, and Ulrike Diebold. Competing stabilization mechanism for the polar ZnO(0001)-Zn surface. Phys. Rev. B, 68: 245409, Dec 2003.

[31] Olga Dulub, Ulrike Diebold, and G. Kresse. Novel stabilization mechanism on polar surfaces: $\mathrm{ZnO(0001)-Zn.} \mathrm{Phys.} \mathrm{Rev.} \mathrm{Lett.,} \mathrm{90:016102,} \mathrm{Jan}$ 2003. 